\title{
Focusing hard $x$ rays to nanometer dimensions using Fresnel zone plates
}

\author{
Christian G. Schroer* \\ HASYLAB at DESY, Notkestrasse 85, D-22607 Hamburg, Germany \\ (Received 7 January 2006; revised manuscript received 27 April 2006; published 14 July 2006)
}

\begin{abstract}
The question is addressed of what is the smallest spot size that hard $\mathrm{x}$ rays can be focused to using Fresnel zone plates. A thick tilted zone plate optic with large numerical aperture is considered in numerical simulations and is shown to efficiently focus hard $\mathrm{x}$ rays down to below $1 \mathrm{~nm}$, well below the theoretical limit for reflective optics such as waveguides and that of refractive optics. The focal spot size is ultimately limited by the atomic structure of matter. The practical realization of these optics will require a significant technological effort, but would enable hard $\mathrm{x}$-ray nanoprobe studies with close to atomic resolution at current and future $\mathrm{x}$-ray sources, such as $\mathrm{x}$-ray free electron lasers and energy recovery linacs.
\end{abstract}

DOI: 10.1103/PhysRevB.74.033405

PACS number(s): 41.50.+h, 07.85.Qe

Since the advent of highly brilliant synchrotron radiation sources, there have been great advances in focusing hard $\mathrm{x}$ rays to ever smaller dimensions. Besides the highly brilliant source, sophisticated $\mathrm{x}$-ray optics are required to generate the smallest foci. In the soft $\mathrm{x}$-ray range, Fresnel zone plates with a diffraction limit below $15 \mathrm{~nm}$ were recently made. ${ }^{1}$ In the hard x-ray range, total reflection mirrors, ${ }^{2}$ multilayer mirrors, ${ }^{3}$ Fresnel zone plates, ${ }^{4}$ multilayer Laue lenses, ${ }^{5}$ waveguides, ${ }^{6}$ and refractive lenses ${ }^{7}$ have all been shown to generate beams with a lateral size well below $100 \mathrm{~nm}$. In all these cases, the focusing underlies technological limits.

Recently, the question of the physical limits to the focusing of $x$ rays arose, in particular whether focusing to atomic dimensions is possible with $\mathrm{x}$ rays. This is of particular interest for the construction of hard $\mathrm{x}$-ray nanoprobes at thirdgeneration synchrotron radiation sources ${ }^{8}$ and future fourthgeneration sources, such as $\mathrm{x}$-ray free electron lasers ${ }^{9}$ and energy recovery linacs. ${ }^{10}$ While the hard $\mathrm{x}$-ray wavelength $\lambda$ is typically on atomic scales and smaller (e.g., $E=20 \mathrm{keV}$ corresponds to $\lambda=0.62 \AA$ ), the diffraction limit of the best optics is currently one to two orders of magnitude larger.

Bergemann et al. ${ }^{11}$ have shown that for optics based on external total reflection, such as waveguides, the numerical aperture is fundamentally limited by the critical angle of external total reflection, $\theta_{\mathrm{c}}=\sqrt{2 \delta}$, resulting in a diffraction limit slightly smaller than $10 \mathrm{~nm}$. In this expression, $\delta$ is the decrement of the index of refraction $(n=1-\delta+i \beta)$ that is limited by the electron density in the reflecting material. Furthermore, they conjectured that this limit holds for all x-ray optics. ${ }^{11}$ While it holds also for thin refractive optics, ${ }^{12}$ this limit can in principle be overcome with thick refractive lenses, so-called adiabatically focusing lenses. ${ }^{13}$ For these optics foci down to about $2 \mathrm{~nm}$ are predicted. In this case, mainly attenuation inside the lens material limits the numerical aperture. Similarly small foci are expected for reflection zone plates. ${ }^{14}$

For diffractive optics such as Fresnel zone plates, the diffraction limit in first order is approximately limited by the width of the outermost zone ${ }^{15}$ that efficiently contributes to image formation. ${ }^{16}$ For hard x rays (e.g., $E=20 \mathrm{keV}$ ), efficient focusing with transmission zone plates requires the optic to be several micrometers thick. To achieve efficient nanometer focusing, aspect ratios of several thousand are needed. One way to realize these structures may be to extract a thin slice out of a laterally and depth-graded multilayer structure, similar to the multilayer-Laue-lens approach described by Kang et al. ${ }^{5}$ Such a structure would focus in one dimension and two such devices would have to be crossed to obtain a point focus. With today's technology, x-ray multilayer optics with layer thicknesses down to $1 \mathrm{~nm}$ can be made. ${ }^{17}$ Using coupled-wave theory efficient focusing with large numerical apertures was shown to require tilted zones that act locally as a diffracting multilayer in Laue geometry. ${ }^{16}$ Since hard $\mathrm{x}$ rays can be diffracted by ordinary matter to large angles, the effect allows one in principle to generate large numerical apertures.

In this article, $\mathrm{x}$-ray wave propagation inside an appropriately tilted thick one-dimensional Fresnel zone plate is considered, simulating the whole optic with the parabolic wave equation in paraxial approximation. Matter is treated as a homogeneous continuum. Within these approximations, the numerical aperture of a thick tilted zone plate is shown to exceed the $\sqrt{2 \delta}$ limit $^{11}$ by an order of magnitude, allowing for efficient focusing to below $1 \mathrm{~nm}$. Wave propagation effects inside an ideal optic do not limit the numerical aperture, as opposed to that in waveguides. ${ }^{11}$ Deviations from the ideal shape may introduce aberrations and lead to reduced efficiency. ${ }^{18,19}$ Eventually, the main fundamental limitation will arise from the atomic structure of matter that will prohibit one from making zone plates with multilayer structures much smaller than 1 nm. ${ }^{17}$

Figure 1 shows the optic considered in this article. This zone plate lens is made of two materials with different refractive indices $n_{1,2}=1-\delta_{1,2}+i \beta_{1,2}$, alternating from one Fresnel zone to the next. At the entrance of the optic, the radius of each zone is given by the zone plate formula $r_{m}(0)=\sqrt{m \lambda f+(m \lambda / 2)^{2}}$, where $m=0, \ldots, M$ is the zone index, $\lambda$ the x-ray wavelength, and $f$ the focal length. Over its thickness $d$, the whole lens shrinks homogeneously by a $z$-dependent factor $a(z)$, such that $r_{m}(z)=r_{m}(0) a(z)$ for all $m=1, \ldots, M$. In principle, for ideal focusing of a plane wave or from point to point, a parabolic or elliptical dependence of $a$ is required, respectively. In practice, however, it turns out that the gain with respect to a linear tilt is marginal if the thickness $d$ is small against the focal length $f$. This is the case even for the small zone plate with its small focal length 


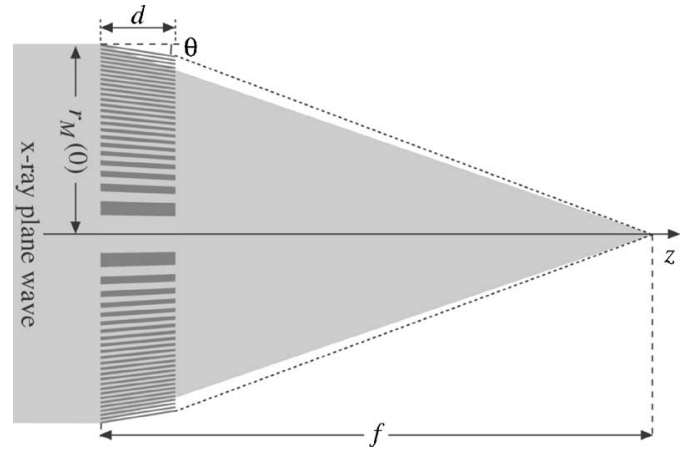

FIG. 1. Focusing geometry for a thick Fresnel zone plate with tilted zones.

considered in this numerical study. To be exact, however, in the following, we consider the focusing of a plane wave with a parabolically tilted zone plate.

For hard $\mathrm{x}$ rays-e.g., with an energy $E=20 \mathrm{keV}$ $(\lambda=0.62 \AA)$-focusing to nanometer dimensions involves small angular deviations from the optical axis, allowing one to use scalar wave equations in paraxial approximation. Freespace propagation of the $\mathrm{x}$ rays is modeled by the FresnelKirchhoff equation that is the paraxial solution to the freespace Helmholtz equation. ${ }^{20}$ Inside the zone plate, propagation along the optical $(z)$ axis is approximated by the parabolic wave equation ${ }^{18,21}$

$$
2 i k \frac{\partial u}{\partial z}+\frac{\partial^{2} u}{\partial x^{2}}+\frac{\partial^{2} u}{\partial y^{2}}+k^{2}\left[n^{2}(x, y, z)-1\right] u=0,
$$

where $k=2 \pi / \lambda$ is the wave vector of the $\mathrm{x}$ rays and $n(x, y, z)=1-\delta(x, y, z)+i \beta(x, y, z)$ is the refractive index inside the optical element. Equation (1) is formally equivalent to the Schrödinger equation, when the $z$ coordinate is interpreted as time. Attenuation inside the material of the optical component is modeled by the imaginary part $i \beta$ of the refractive index and makes the optical potential complex, generating a sink for the amplitude $u$ along the propagation direction. With attenuation included, the operator $\hat{H}=\frac{\partial^{2}}{\partial x^{2}}+\frac{\partial^{2}}{\partial y^{2}}+k^{2}\left[n^{2}(x, y, z)-1\right]$ is non-Hermitian.

The solution to this equation can be easily constructed for optical structures that are invariant along the optical axisi.e., $\partial n / \partial z=0$ - such as the regular Fresnel zone plate without tilted zones. In that case, Eq. (1) does not explicitly depend on $z$. The incident wave field $\psi_{0}(x, y)$ can be expressed in a complete set of transverse (forward) eigenmodes $\left|\psi_{0}\right\rangle=\Sigma_{n} C_{n}\left|\phi_{n}\right\rangle$, where $C_{n}=\left\langle\phi_{n} \mid \psi_{0}\right\rangle$. Propagation through the optic is given by multiplication of each eigenmode $\left|\phi_{n}\right\rangle$ with an appropriate phase factor $\exp \left\{-i k \lambda_{n} d\right\}$, where $\lambda_{n}$ is the complex eigenvalue of $\left|\phi_{n}\right\rangle$ with respect to $\hat{H}$. The wave field exiting the optic is given by $\left|\psi_{d}\right\rangle=\Sigma_{n} C_{n} \exp \left\{-i k \lambda_{n} d\right\}\left|\phi_{n}\right\rangle$. In case the structures of the optic are not invariant along the optical axis-e.g., the tilted zone plate in Fig. 1-the eigensystem changes as a function of $z$. In that case, the wave field at a position $z$ can be expressed as

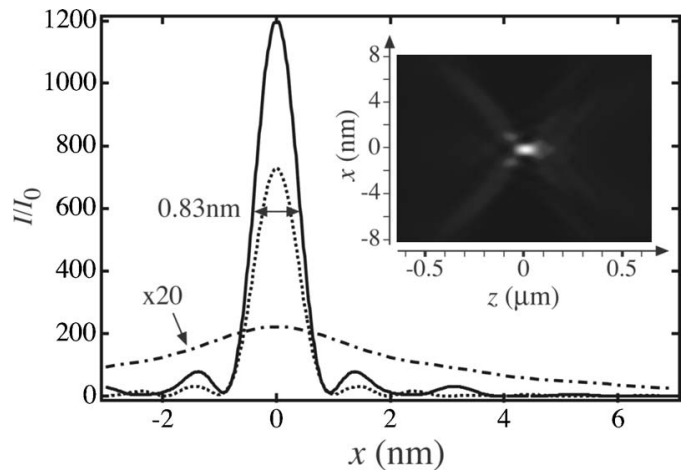

FIG. 2. First-order focus of a parabolically tilted zone plate (solid line), an equivalent ideal thin zone plate (dotted line), and a straight (not tilted) zone plate (dash-dotted line). The inset shows the beam intensity distribution around the focus along the optical axis for the parabolically tilted zone plate.

$$
\left|\psi_{z}\right\rangle=\sum_{n} C_{n}(z) \exp \left\{-i k \int \lambda_{n}(z) d z\right\}\left|\phi_{n}(z)\right\rangle .
$$

The evolution of the coefficients $C_{n}(z)$ can be determined by inserting Eq. (2) into Eq. (1), leading to the system of ordinary differential equations

$$
\frac{\partial}{\partial z} C_{k}=-\sum_{n} C_{n}\left\langle\phi_{k} \mid \frac{\partial}{\partial z} \phi_{n}\right\rangle \exp \left\{-i k \int\left(\lambda_{n}-\lambda_{k}\right) d z\right\},
$$

which can be solved numerically, if the transition matrix $\left\langle\phi_{k} \mid \frac{\partial}{\partial z} \phi_{n}\right\rangle$ and the eigenvalues and eigenfunctions are known as a function of $z$. In the case of a tilted zone plate (cf. Fig. 1), the eigenvalues and functions can be calculated numerically for different positions along the optic. These calculations show that the eigenvalues follow a scaling law and the eigenfunctions are nearly unchanged [for a small range of $a(z)$ ] with respect to coordinates rescaled by $a(z)$. In this way, the eigensystem can be interpolated during the integration of Eq. (3).

Consider now a tilted one-dimensional zone plate made of $\mathrm{Ni}$ (separated by vacuum) as shown in Fig. 1 with $N=400$ zones $\left[r_{1}(0)=40 \mathrm{~nm}\right]$. In this geometry, the outermost zone has a width of $\Delta r_{M}(0)=1.00 \mathrm{~nm}$. The aperture of this optic is $1.60 \mu \mathrm{m}$. This very small zone plate was chosen to be able to perform a full parabolic wave equation calculation for one transverse dimension (one-dimensional focusing) at $2 \AA$ resolution. ${ }^{22}$ The material $\mathrm{Ni}$ was chosen to make $d$ small enough with respect to $f$. In a more practical situation, a zone plate with the same numerical aperture would be made of an appropriate multilayer system and have many more zones, a much larger aperture, and a larger focal distance. ${ }^{5}$

The transmission of a hard $\mathrm{x}$-ray plane wave $(E=20 \mathrm{keV})$ through the tilted zone plate with a thickness of $d=8 \mu \mathrm{m}$ was calculated by numerically integrating Eq. (3) using a Runge-Kutta method. ${ }^{23}$ Behind the optic, the resulting wave field was propagated to the focal plane using the Fresnel-Kirchhoff equation. Figure 2 shows the transverse beam profile in the focus for a tilted thick zone plate tuned to 
the optimal first-order Bragg condition of the outermost zones. ${ }^{24}$ As a result of refraction inside the optic, the focus is found at $f=25.73 \mu \mathrm{m}$ from the entrance of the optic, slightly closer than for the thin case $\left(f_{\text {thin }}=25.8 \mu \mathrm{m}\right)$. The diffraction limited full width at half maximum (FWHM) beam size is $d_{\mathrm{t}}=0.87 \mathrm{~nm}$ and the Rayleigh criterion yields $d_{R}=0.93 \mathrm{~nm}$, being slightly smaller than that expected for an ideal thin zone plate $\left(d_{R(\text { thin })}=1.00 \mathrm{~nm}\right.$, dotted curve in Fig. 2$){ }^{25}$ The thickness of the zone plate $(d=8 \mu \mathrm{m})$ was chosen such that the diffracted mode is optimally excited and multiple scattering inside the lens is negligible. If the lens is made thicker, the efficiency and focus quality decrease as multiple scattering becomes relevant. In the given example, $63.1 \%$ of the radiation that enters the lens aperture is focused in one dimension, showing that efficient focusing at large numerical apertures is possible. As opposed to a thin zone plate, only the diffraction order fulfilling the Bragg condition is efficiently excited, resulting in the increased efficiency as compared to the ideal thin zone plate (dotted line in Fig. 2). The dash-dotted curve in Fig. 2 shows the intensity distribution for a straight (not tilted) zone plate, illustrating that indeed propagation effects inside this optic dramatically reduce the diffraction efficiency of the outermost zones.

The sensitivity of efficient focusing to angular and energy deviations depends on the Darwin width of the diffraction peak of the outermost zones. In the given case, monochromatic radiation from an interval of about $100 \mathrm{eV}$ or angular deviations up to $50 \mu \mathrm{rad}$ are accepted by the optic. While this limited field of view is sufficient to focus a thirdgeneration undulator source onto a sample without loss of efficiency and resolution, the optic is not suited to image extended objects in full field microscopy.

The small focus size suggests that the outermost zones contribute to the focus. This can be examined in more detail by investigating the wave field inside the optic. Figure 3 shows the field intensity inside the zone plate structure. As a superposition of the transmitted and focused beam a standing wave is formed along the zone boundaries (cf. Fig. 3). This standing wave is created in analogy to that by Laue diffraction inside a crystal and persists up to the aperture of the zone plate [Fig. 3(c)]. As a measure for the local efficiency of the zone plate the flux density in the (transverse) $x$ direction,

$$
J_{x}(x, z)=\frac{1}{2 i k}\left[\left\langle\psi \mid \partial_{x} \psi\right\rangle-\left\langle\partial_{x} \psi \mid \psi\right\rangle\right],
$$

can be examined. It is a measure for the flow of photons perpendicular to the optical axis. Figure 4 shows $J_{x}(x, d)$ at the exit of the zone plate compared to that of an ideal converging spherical wave with the same focused intensity and bounded by the numerical aperture given by the gray shaded cone in Fig. 1. The flux density oscillates with the zone structure and grows on average linearly with distance from the optical axis, similarly to that of the converging spherical wave. Toward the edge of the optic, the diffraction efficiency drops. The drop in efficiency is a result of the finiteness of the grating both perpendicular to and along the grating lines. The smooth drop-off takes place on the extinction length of

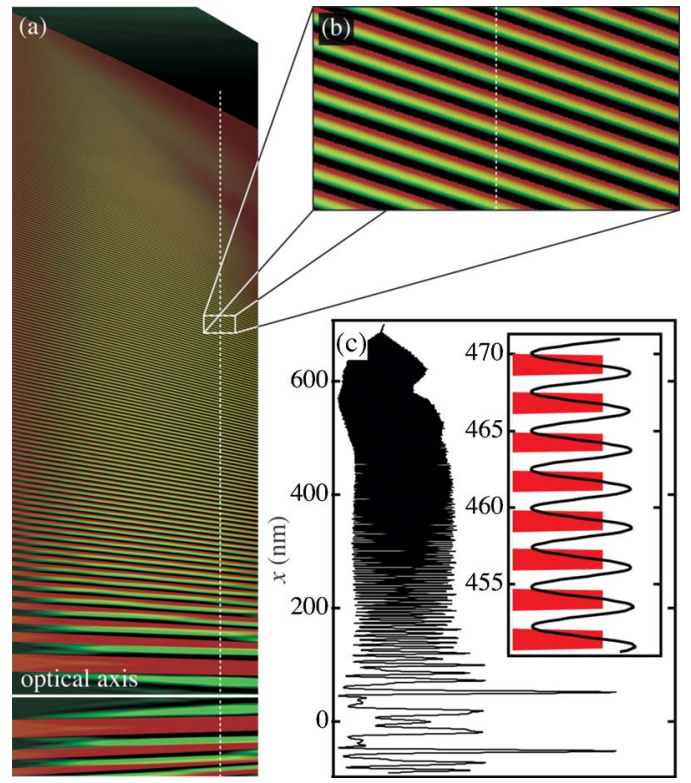

FIG. 3. (Color online) (a) X-ray intensity (green-light gray) inside a tilted zone plate focusing in first order. The Ni zones are shown in red (dark gray). Slightly more than half of the zone plate is depicted in (a). (b) Enlargement of the rectangular region in (a) showing the formation of the standing wave along the zone boundaries. (c) Intensity along the dashed line in (a). In the inset in (c) the intensity along the dashed line in (b) is shown together with the zone plate structure (red-gray solid bars).

the grating. ${ }^{26}$ The dip in both the wave intensity [Fig. 3(c)] and flux (Fig. 4) at slightly below $600 \mathrm{~nm}$ from the center of the lens is due to the abrupt limits of the zone plate and the resulting lack of diffracted amplitude from beyond the aperture. These finite optic effects define the diffraction limit and exact shape of the focus and cannot be obtained from coupled wave theory that assumes infinitely extended gratings.

The reduction in diffraction efficiency toward the edge of the optic explains why it does not have the maximal numerical aperture given by its geometry (cone delimited by the dashed line in Fig. 1). It turns out that effectively the numerical aperture is close to that of an ideal thin optic corresponding to the gray shaded cone in Fig. 1.

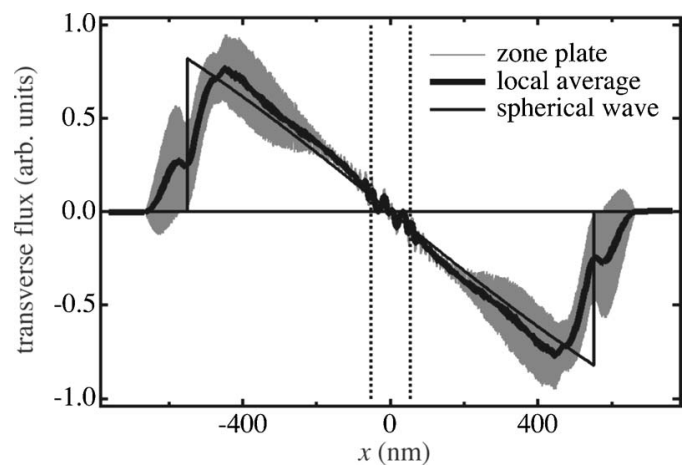

FIG. 4. Transverse flux at the exit of the optic $(z=d)$ compared to that of a spherical wave with numerical aperture $r_{M}(0) / f$. The aperture between the dashed vertical lines corresponds to the $\sqrt{2 \delta}$ limit. 
The first-order diffraction requires the fabrication of structures on the order of the spatial resolution to be achieved. To relax the requirements on the fabrication while keeping the spatial resolution constant or to increase the spatial resolution, higher-order reflections can be excited. To demonstrate this scheme, a parabolically tilted $\mathrm{Ni}$ zone plate with $N=120$ zones $\left[r_{1}(0)=60 \mathrm{~nm}\right]$ and an outermost zone width of $r_{M}(0)=2.74 \mathrm{~nm}$ is investigated. The tilt is set to excite the third-order reflection. For this optic, a FWHM beam size of about $0.7 \mathrm{~nm}$ is obtained. The Rayleigh criterion yields $0.75 \mathrm{~nm}$ resolution, being slightly better than that for the idealized thin zone plate. However, compared to the first order, the third-order diffraction is significantly less efficient (by about one order of magnitude) and will be more sensitive to imperfections. The reduction in efficiency can to some extent be compensated for by adapting the line-to-space ratio of the optic, ${ }^{27}$ requiring in turn the generation of smaller structures. For optimal focusing in this case, an appropriate trade-off between minimal feature size and diffraction efficiency has to be found.

I would like to thank F. Pfeiffer, I. Vartaniants, E. Weckert, and B. Lengeler for helpful discussions.
*Present address: Inst. of Structural Physics, Dresden University of Technology, D-01062 Dresden, Germany.

${ }^{1}$ W. Chao, B. D. Harteneck, J. A. Liddle, E. H. Anderson, and D. T. Attwood, Nature (London) 435, 1210 (2005).

${ }^{2}$ H. Mimura, S. Matsuyama, H. Yumoto, K. Yamamura, Y. Sano, M. Shibahara, K. Endo, Y. Mori, Y. Nishino, K. Tamasaku, M. Yabashi, T. Ishikawa, and K. Yamauchi, Jpn. J. Appl. Phys., Part 2 44, L539 (2005).

${ }^{3}$ O. Hignette, P. Cloetens, G. Rostaing, P. Bernard, and C. Morawe, Rev. Sci. Instrum. 76, 063709 (2005).

${ }^{4}$ http://www.xradia.com

${ }^{5}$ C. Liu, R. Conley, A. T. Macrander, J. Maser, H. C. Kang, M. A. Zurbuchen, and G. B. Stephenson, J. Appl. Phys. 98, 113519 (2005); H. C. Kang, G. B. Stephenson, C. Liu, R. Conley, A. T. Macrander, J. Maser, S. Bajt, and H. N. Chapman, Appl. Phys. Lett. 86, 151109 (2005); H. C. Kang, J. Maser, G. B. Stephenson, C. Liu, R. Conley, A. T. Macrander, and S. Vogt, Phys. Rev. Lett. 96, 127401 (2006).

${ }^{6}$ A. Jarre, C. Fuhse, C. Ollinger, J. Seeger, R. Tucoulou, and T. Salditt, Phys. Rev. Lett. 94, 074801 (2005).

${ }^{7}$ C. G. Schroer, O. Kurapova, J. Patommel, P. Boye, J. Feldkamp, B. Lengeler, M. Burghammer, C. Riekel, L. Vincze, A. van der Hart, and M. Küchler, Appl. Phys. Lett. 87, 124103 (2005).

${ }^{8}$ Hard $\mathrm{x}$-ray scanning microscope stations are planned at ESRF and APS [J. Maser, G. B. Stephenson, D. Shu, B. Lai, S. Vogt, A. Khounsary, Y. Li, C. Benson, and G. Schneider, in Synchrotron Radiation Instrumentation, edited by T. Warwick, J. Arthur, H. A. Padmore, and J. Stöhr, AIP Conf. Proc. No. 705 (AIP, Melville, NY, 2004), p. 470].

${ }^{9}$ Several hard x-ray free electron laser projects are currently being pursued (cf., e.g., http://www-ssrl.slac.stanford.edu/lcls/, http:// xfel.desy.de).

${ }^{10} \mathrm{~A}$ hard $\mathrm{x}$-ray energy recovery linac is currently being developed at Cornell University [D. H. Bilderback et al., J. Synchrotron Radiat. 10, 346 (2003)].

${ }^{11}$ C. Bergemann, H. Keymeulen, and J. F. van der Veen, Phys. Rev. Lett. 91, 204801 (2003).
${ }^{12}$ Y. Suzuki, Jpn. J. Appl. Phys., Part 1 43, 7311 (2004).

${ }^{13}$ C. G. Schroer and B. Lengeler, Phys. Rev. Lett. 94, 054802 (2005).

${ }^{14}$ A. G. Michette, S. J. Pfauntsch, A. Erko, A. Firsov, and A. Svintsov, Opt. Commun. 245, 249 (2005).

${ }^{15}$ J. Kirz, J. Opt. Soc. Am. 64, 301 (1974).

${ }^{16} \mathrm{~J}$. Maser, in X-Ray Microscopy IV, edited by V. V. Aristov and A. I. Erko (Institute of Microelectronics Technology, Chernogolovka, Russia, 1994), pp. 523-530.

${ }^{17}$ C. Liu, R. Conley, A. T. Macrander, T. Graber, C. Morawe, C. Borel, and E. M. Dufresne, Proc. SPIE 5537, 154 (2004).

${ }^{18}$ A. N. Kurokhtin and A. V. Popov, J. Opt. Soc. Am. A 19, 315 (2002).

${ }^{19}$ G. Schneider, Appl. Phys. Lett. 73, 599 (1998).

${ }^{20} \mathrm{M}$. Born and E. Wolf, Principles of Optics (Cambridge University Press, Cambridge, England, 1999).

${ }^{21}$ Bergemann et al. (Ref. 11) use this very same model to treat the $\mathrm{x}$ rays inside a waveguide.

${ }^{22}$ The wave field in this zone plate was represented numerically by 9216 complex eigenmodes in a field of view of about $1.84 \mu \mathrm{m}$ centered around the optical axis. Periodic boundary conditions were used at the boundary of the transverse region.

${ }^{23}$ W. H. Press, S. A. Teukolsky, W. T. Vetterling, and B. P. Flannery, Numerical Recipes in $C$ (Cambridge University Press, Cambridge, England, 1992).

${ }^{24}$ In multilayer systems, refraction effects can lead to significant deviations from the Bragg condition $n \lambda=2 d \sin \theta$ towards larger angles (Ref. 26). In the present example, the Bragg angle must be increased by about $1 \%$ to yield optimal performance.

${ }^{25}$ The diffraction limit for one-dimensional focusing is given by $d_{\mathrm{R}}=\lambda /(2 N A)$ [G. O. Reynolds, J. B. DeVelis, G. B. Parrent, Jr., and B. J. Thompson, Physical Optics Notebook: Tutorials in Fourier Optics (SPIE Optical Engineering Press, Bellingham, WA, 1989)].

${ }^{26}$ J. Underwood and T. Barbee, Jr., Appl. Opt. 20, 3027 (1981).

${ }^{27}$ G. Schneider, Appl. Phys. Lett. 71, 2242 (1997). 